\title{
História do Grupo de Trabalho em Medicina Rural da SBMFC
}

\section{History of the SBMFC Working Party on Rural Medicine}

\section{Historia del Grupo de Trabajo de Medicina Rural SBMFC}

\author{
Leonardo Vieira Targa $^{1}$, Mayara Floss ${ }^{2 \oplus}$, Gustavo Gusso ${ }^{3 \oplus}$, Magda Almeida ${ }^{4}$, Nilson Massakazu Ando ${ }^{5 \oplus}$, Monica Lima ${ }^{6 \oplus}$, \\ André Luiz da Silva ${ }^{7}$ \\ ${ }^{1}$ Universidade de Caxias do Sul, Caxias do Sul, RS, Brasil. \\ ${ }^{2}$ Residente em Medicina de Família e Comunidade do Grupo Hospitalar Conceição, RS, Brasil. \\ ${ }^{3}$ Universidade de São Paulo, São Paulo, SP, Brasil. \\ ${ }^{4}$ Universidade Federal do Ceará, CE, Brasil. \\ ${ }^{5}$ UN-AM/Petrobras e Grupo Especial de Supervisão/MEC. \\ ${ }^{6}$ Prefeitura Municipal de Cajati, SP, Brasil. \\ ${ }^{7}$ Pontifícia Universidade Católica do Rio Grande do Sul, Brasil.
}

\section{Resumo}

Este artigo apresenta a História do Grupo de Trabalho em Medicina Rural da SBMFC, um dos mais ativos grupos de trabalho da SBMFC. São revisados os primeiros passos desde a sua criação e posteriores desenvolvimentos. Um dos principais resultados identificados foi o de ajudar a consolidar a identidade do "MFC rural" e trazer a discussão da saúde das populações rurais para a agenda da saúde, da atenção primária e da medicina de família. Este relato tem também como objetivo auxiliar outros grupos em criação ou já estabelecidos a criar estratégias de fortalecimento de seus trabalhos.

Palavras-chave: Saúde da População Rural; Medicina de Família e Comunidade; História da Medicina .

\begin{abstract}
This article presents the History of the SBMFC Working Party on Rural Medicine, one of SBMFC's most active working groups. The first steps since its creation and further developments are reviewed. One of the main results identified was to help consolidate the identity of the "rural family doctor" and bring the discussion of the health of rural populations to the health, primary care and family medicine agenda. This report also aims to assist other groups in creation or already established to create strategies to strengthen their work.
\end{abstract}

Keywords: Rural Health; Family Practice; History of Medicine.

Como citar: Targa LV, Floss M, Gusso G, Almeida M, Ando NM, Lima M, et al. História do Grupo de Trabalho em Medicina Rural da SBMFC. Rev Bras Med Fam Comunidade. 2020;15(42):2365. https://doi.org/10.5712/rbmfc15(42)2365
Autor correspondente: Leonardo Vieira Targa. E-mail: targalv@gmail.com Fonte de financiamento: declaram não haver. Parecer CEP: não se aplica. Recebido em: 29/01/2020. Aprovado em: 23/03/2020. 


\section{Resumen}

Este artículo presenta la Historia del Grupo de Trabajo de Medicina Rural de SBMFC, uno de los grupos de trabajo más activos de SBMFC. Se revisan los primeros pasos desde su creación y otros desarrollos. Uno de los principales resultados identificados fue ayudar a consolidar la identidad del "MFC rural" y llevar el debate sobre la salud de las poblaciones rurales a la agenda de salud, atención primaria y medicina familiar. Este informe también tiene como objetivo ayudar a otros grupos en la creación o ya establecidos para crear estrategias para fortalecer su trabajo. Descriptores: Salud rural, Medicina Familiar y Comunitaria, Historia de La Medicina.

Palabras clave: Salud Rural; Medicina Familiar y Comunitaria; Historia de la Medicina.

\section{INTRODUÇÃO}

A Sociedade Brasileira de medicina de Família e Comunidade (SBMFC) define grupo de trabalho (GT) como "um grupo de Médicos de Família e Comunidade e colaboradores reconhecidos pela SBMFC com o objetivo de apoiar a assistência, a educação permanente e o desenvolvimento profissional continuado, a crítica científica e a pesquisa, pelos profissionais da Atenção Primária à Saúde (APS); a formação de estudantes de Áreas de Saúde; e o empoderamento da comunidade quanto à sua saúde."1

O GT de Medicina Rural é um dos mais antigos e um dos mais ativos e produtivos grupos dessa sociedade. Este trabalho tem como objetivo descrever a história deste grupo desde sua formação até os dias atuais. Com isso, busca-se suprir uma demanda de outros grupos ainda em formação sobre os passos importantes para a formação de um grupo forte e produtivo além do registro de uma parte importante da história da SBMFC. Este conhecimento ajuda a compreender o estágio atual do trabalho da Sociedade e do GT rural e planejar novos movimentos dentro dos objetivos estratégicos dos mesmos.

\section{Primeiros Passos}

Os primeiros acontecimentos importantes para a mobilização de médicos de família e comunidade (MFC) em torno da temática rural foram claramente ligados ao movimento de saúde rural internacional (foto 1), mais especificamente relacionados à Associação Mundial de Médicos de Família (WONCA) e seu muito ativo Working Party on Rural Health (WWPRP). Os principais passos desta história podem ser visualizados na Tabela 1. Até o início da década de 2010, os estágios rurais que ocorrem no internato de algumas faculdades de medicina eram a principal experiência com o tema no Brasil, mas de maneira dispersa e pouco estruturada.

Em 2009, alguns MFCs que trabalhavam em áreas rurais e começavam a desenvolver interesse por relações com ensino e pesquisa, foram estimulados pela SBMFC a participaram de dois eventos internacionais importantes: (1) o 17th International Congress of Agricultural Medicine \& Rural Health organizado pela International Association of Agricultural Medicine and Rural Health e pelo governo Colombiano; e, (2) a 9th WONCA Rural Health World Conference. Nesses dois eventos, foram feitos os primeiros contatos com membros do WWPRP, o que futuramente seria um grande estimulador dos trabalhos nacionalmente, como veremos adiante. No primeiro evento, na Colômbia, os MFCs Nilson Ando e Ricardo Amaral Filho apresentaram trabalhos relacionados às suas experiências na região Amazônica, com populações ribeirinhas, incluindo o uso da telemedicina em locais de difícil acesso e Leonardo Vieira 
Targa apresentou na Grécia resultados do rastreamento de hipertensão arterial sistêmica em regiões rurais, por meio de protocolo que envolvia o treinamento de agentes comunitários de saúde. ${ }^{2}$

Ainda neste ano, os três participaram da primeira atividade conjunta com membros do WWPRP, no caso o Dr. Roger Strasser e membros da SBMFC, que ainda não tinha grupos de trabalho organizados. Após a palestra dos três participantes, houve uma pequena oficina aberta a interessados, onde a experiência rural local dos participantes dialogou com a rica construção canadense da Northern Ontario School of Medicine. Diversos trabalhos relacionados à medicina rural foram apresentados já neste evento, o $10^{\circ}$ Congresso Brasileiro de Medicina de Família e Comunidade, entre eles uma avaliação do estágio de medicina de família rural de Nova Petrópolis (RS) e a experiência no atendimento em uma Unidade Móvel Fluvial, em Manaus (AM) e em Santarém (PA), que serviriam de base para que o Ministério da Saúde desenvolvesse financiamentos específicos para Equipes de Saúde Ribeirinhas e para a construção de Unidades Básicas de Saúde Fluvial (UBSF).

No ano seguinte, ocorreu a primeira participação de um membro da SBMFC em uma reunião específica do WWPRP, que ocorreu durante a 19th WONCA World Conferenceof Family Doctors, no México, onde também foi apresentada a palestra com o título Empowering Rural Health in Central and South America: the role of primary care and familiy medicine. Neste evento, ocorreram as primeiras negociações para uma futura apresentação do Brasil como futuro país sede de uma conferência mundial de medicina rural.

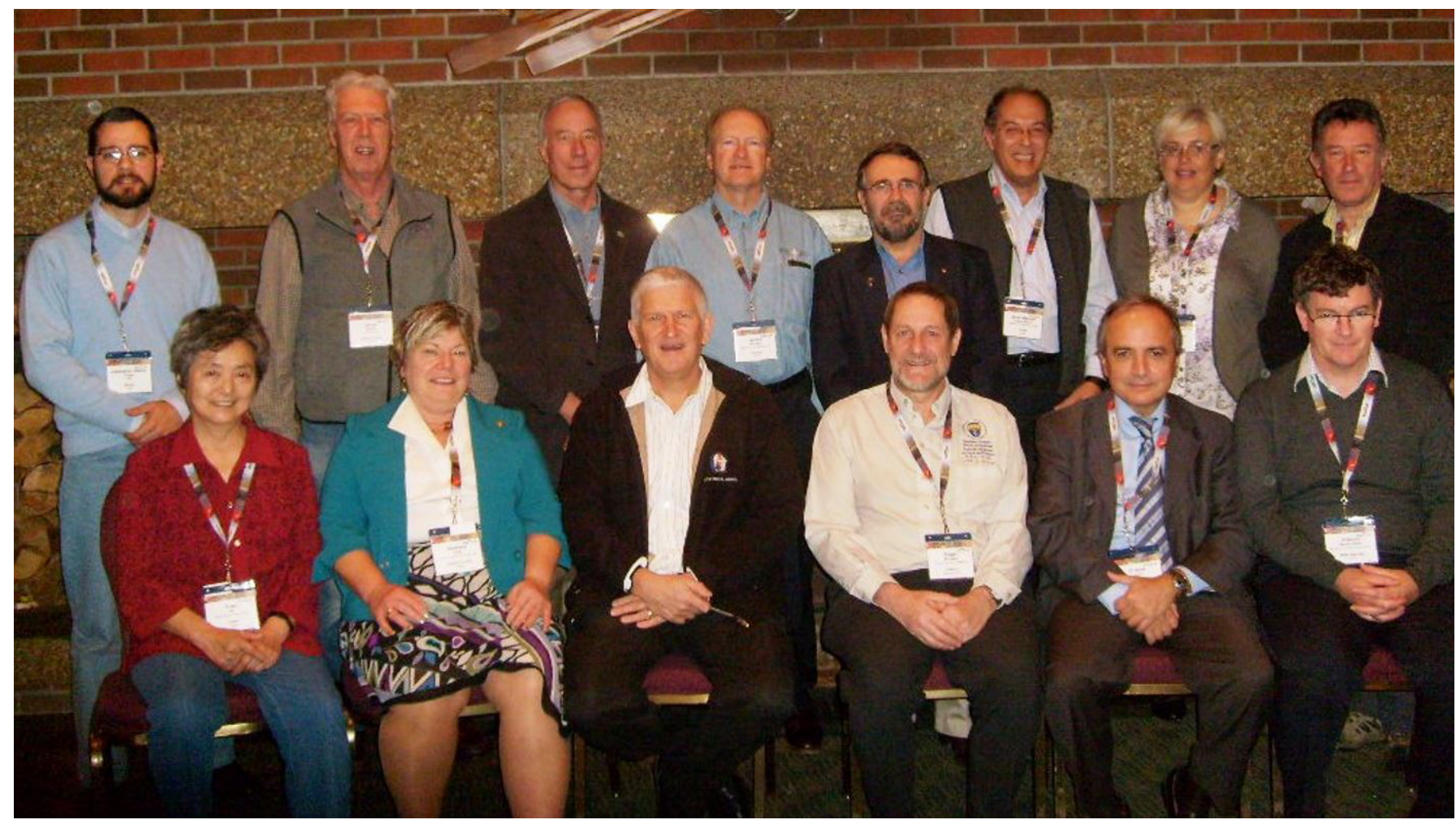

Foto 1. Reunião do WONCA Working Party on Rural Practice, em ThunderBay, 2012. A parceria entre SBMFC e WONCA foi importante desde o início para a formação e desenvolvimento do GT rural. 
Ainda em 2010, trabalhos e palestras foram apresentados pela primeira vez em congressos regionais da SBMFC: o II Congresso Sul-Brasileiro de MFC, em Curitiba, e o V Congresso Mineiro de Medicina de Família e Comunidade, que ocorreu simultaneamente ao I Simpósio Centro-Sul Brasileiro em MFC.

Estes passos foram fundamentais para o amadurecimento da ideia de criar um GT rural, contribuindo para a formação de uma identidade de médicos de família que também são médicos rurais, a partir do entendimento das especificidades da prática da MFC nestas áreas e a criação de laços pessoais e institucionais em diversos níveis (regionais, nacionais e internacionais). O conhecimento da realidade mundial, muito variada e diversa da que estávamos habituados, gerou um despertar gradual para as necessidades nacionais e as contribuições que poderíamos fazer a partir de nossas experiências, que estavam acontecendo tanto na região rural do Sul do Brasil quanto na região Amazônica. O apoio da gestão 2010-2012 da SBMFC foi fundamental para que este conjunto de fatores fosse tão bem articulado na figura posterior do GT rural e que tem gerado tão bons frutos. Por causa do tamanho continental e da necessidade de fixar médicos em áreas consideradas remotas, a medicina rural foi entendida desde essa gestão a área de atuação mais importante da MFC. Este aspecto aliado à necessidade de se estruturar os grupos de trabalho fez da medicina rural o motor deste movimento dentro da SBMFC. Um dos resultados foi a criação de um cargo específico de diretor de medicina rural na estrutura administrativa da entidade.

\section{Criação do GT rural}

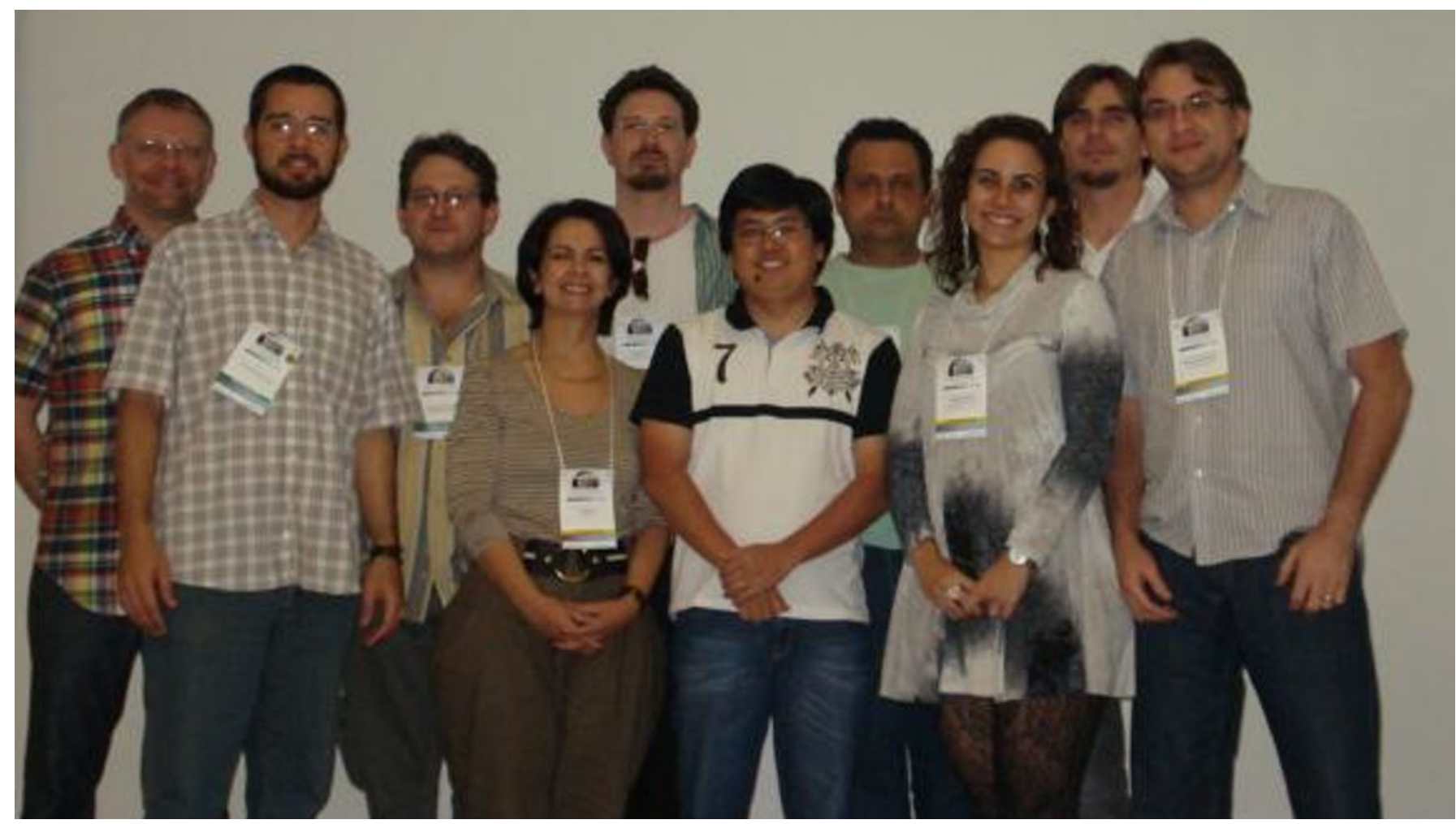

Foto 2. Primeira reunião do GT rural, em Brasília, 2011. Da esquerda para a direita: Marcos Breunig, Leonardo Vieira Targa, Leonardo Savassi, Mônica Lima, Enrique Barros, Nilson Ando, Dijon Hosana, Thereza Horta, Fábio Schwalm e Arnaldo Almeida. 
A criação propriamente dita do GT rural deu-se em 2011, durante o $11^{\circ}$ Congresso Brasileiro de Medicina de Família e Comunidade, ocorrido em Brasília (foto 2). Em reunião de um dia inteiro, paralelamente à programação do evento, que também contou com trabalhos relacionados ao tema por membros do futuro grupo, estabeleceu-se a visão norteadora "EXCELÊNCIA EM SAÚDE RURAL PARA TODAA POPULAÇÃO NO BRASIL" e a nossa missão enquanto grupo: "PROMOVER AQUALIFICAÇÃO E A EXCELÊNCIA DA SAÚDE RURAL NO BRASIL, RESPEITANDO A DIVERSIDADE DE CADA REGIÃO".

Também foram listados objetivos:

- Tratar das questões de interiorização da Medicina de Família e Comunidade.

- Estimular o uso de tecnologias apropriadas para superar barreiras geográficas.

- Estimular a produção de conhecimentos sobre a Medicina Rural.

- Criar uma rede colaborativa de saúde rural inter-regional.

- Fomentar, junto às Instituições de Ensino Superior, a inserção da saúde rural nos currículos de graduação.

- Estabelecer parcerias com Programas de Residência em Medicina de Família e Comunidade, Residência Multiprofissional, Especialização, Mestrado e Doutorado de instituições que possibilitem o desenvolvimento da saúde rural no Brasil.

- Fomentar os processos de desenvolvimento profissional continuado específico para profissionais que trabalhem em área considerada rural.

- Estimular a troca de experiências nacionais e internacionais sobre a saúde rural.

- Estabelecer parcerias, de forma interdisciplinar e intersetorial, com grupos ou entidades, para promover a atenção a saúde nas comunidades rurais.

- Desenvolver estratégias para o recrutamento, a contratação e a fixação de médicos rurais no Brasil.

Participaram deste momento fundante os seguintes colegas: Nilson Ando (Manaus/AM) Diretor de Medicina Rural da SBMFC, Leonardo Vieira Targa (Nova Petrópolis/RS) coordenador do GT e da reunião, Arnaldo Almeida (Divinópolis/MG), Dijon Hosana (Ilhéus/BA), Enrique Barros (Santa Maria do Herval/RS), Fábio Schwalm (Barão/RS), Leonardo Savassi (Ouro Preto/MG), Marcos Breunig (Ajuricaba/RS), Mônica Lima (Cajati/SP), Ricardo Amaral Filho (Manaus/AM), e Thereza Horta (Brumadinho/MG).

Foram discutidas e pactuadas as formas de funcionamento do GT, visto que não havia precedentes para servir de modelo na SBMFC, e decidido que o grupo funcionaria de forma aberta, porém mantendo uma separação entre membros e colaboradores, em parte inspirado na forma de funcionamento do WWPRP. Os membros seriam MFCs sócios adimplentes e teriam direito a voto e ocupar cargos no GT. Estas regras posteriormente ajudaram a SBMFC a definir sua norma geral para GTs.

Neste evento ainda se apresentou a aprovação recente do Brasil como sede da primeira edição na América Latina do Congresso Mundial de Saúde Rural da WONCA, então planejada para ocorrer em 2014. Esta ocorrera recentemente no Congresso de Sinaia, Romênia, onde Leonardo Targa apresentou oficialmente a proposta já esboçada anteriormente no México e contando também com o reforço em Cebu, Filipinas, por parte de Nilson Ando. Alinhavou-se a divulgação regional deste em Santa Fé, Argentina, onde ocorreria em breve um Foro Regional de Expertos em Salud Rural e para os futuros eventos nacionais e regionais. A perspectiva de um evento deste porte foi possível também pela parceria com a Associação 


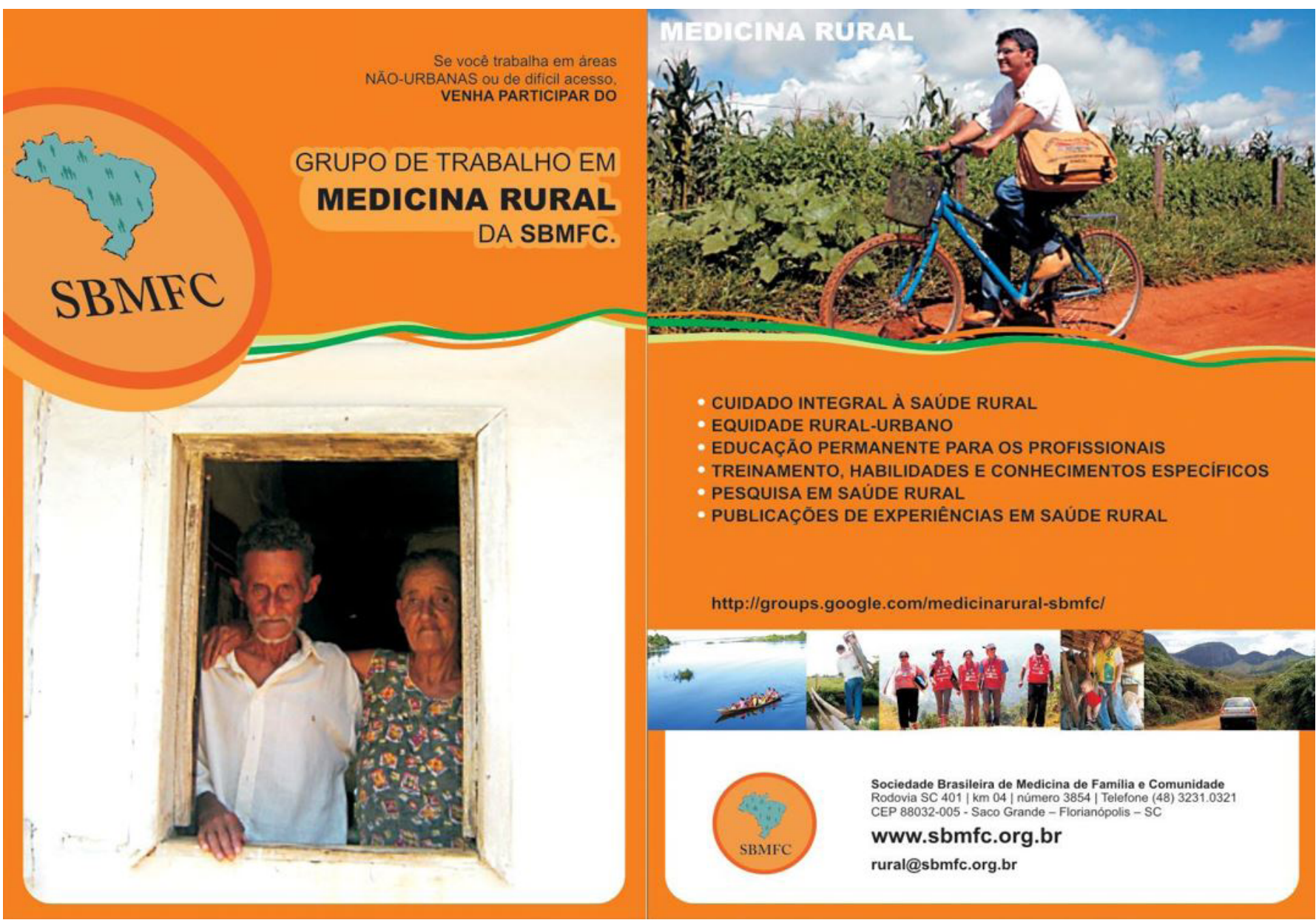

Foto 3.Primeiro folder de divulgação do GT rural, com fotos tiradas por MFCs rurais em suas práticas diárias.

Gaúcha de Medicina de Família e Comunidade, na época liderada pelos colegas José Mauro Ceratti Lopes e Luiz Felipe Mattos, cujo apoio foi essencial.

Em Brasília, também foram acordadas publicações em capítulos do Programa de Atualização em Medicina de Família e Comunidade (PROMEF) da ARTMED, bem como a inserção do tema de saúde rural nos principais livros de Medicina Ambulatorial e de MFC do país, como forma de iniciarmos uma identidade de publicação ao redor da saúde rural. Combinou-se ainda uma publicação com as experiências dos colegas do GT, que alguns anos mais tarde consolidaram-se em um livro ilustrado.

Outra atividade importante deste primeiro encontro do GT rural foi a divisão de temas de interesse entre os membros do grupo, que alinhavaram diversas atividades posteriores como, por exemplo, saúde de populações ribeirinhas, quilombolas, indígenas, uso de ferramentas de telessaúde para equipes rurais, clima, saúde rural, etc. O primeiro material de divulgação do GT, que foi então pactuado, pode ser visualizado na foto 3 .

A discussão de parcerias importantes fora da SBMFC também foi estabelecida como meta e geraram posteriores desdobramentos com o Ministério da Saúde, Associação Brasileira de Saúde Coletiva, outras sociedades de saúde e medicina rural internacionais e universidades. 
O marco final desta reunião fundadora foi o estabelecimento de um consenso em torno da produção do primeiro documento do GT rural, a Declaração de Brasília, ${ }^{3}$ onde a discussão do conceito de ruralidade foi revista à luz da experiência dos integrantes do GT rural e em relação às especificidades da MFC.

\section{Desdobramentos}

Após este momento fundacional, várias ações foram desencadeadas, como a inserção de capítulos sobre medicina rural nos principais livros de medicina ambulatorial do país (como o Duncan e o Tratado de Medicina de Família e Comunidade), bem como no PROMEF, traduções dos principais documentos internacionais na área para o português. ${ }^{4}$ Ao mesmo tempo, a articulação nacional e internacional prosseguia com participação de membros do GT nos congressos do WWPRP em diversos países, bem como nos congressos nacionais e regionais da SBMFC e em outros eventos relacionados. Esta presença foi importante, entre outros aspectos, para dar prosseguimento a preparação da XII Conferência Mundial de Saúde Rural da WONCA, primeira vez em que o evento foi realizado na América Latina. A responsabilidade de organizar tal evento foi um dos grandes motivadores para a criação e mobilização do grupo nestes anos iniciais.

A Conferência Mundial de Saúde Rural ocorreu de $1^{\circ}$ a 6 de abril de 2014, em Gramado (RS), e reuniu mais de 700 participantes vindos de mais de 20 países, incluindo autoridades mundiais na área (foto 4$).^{5}$

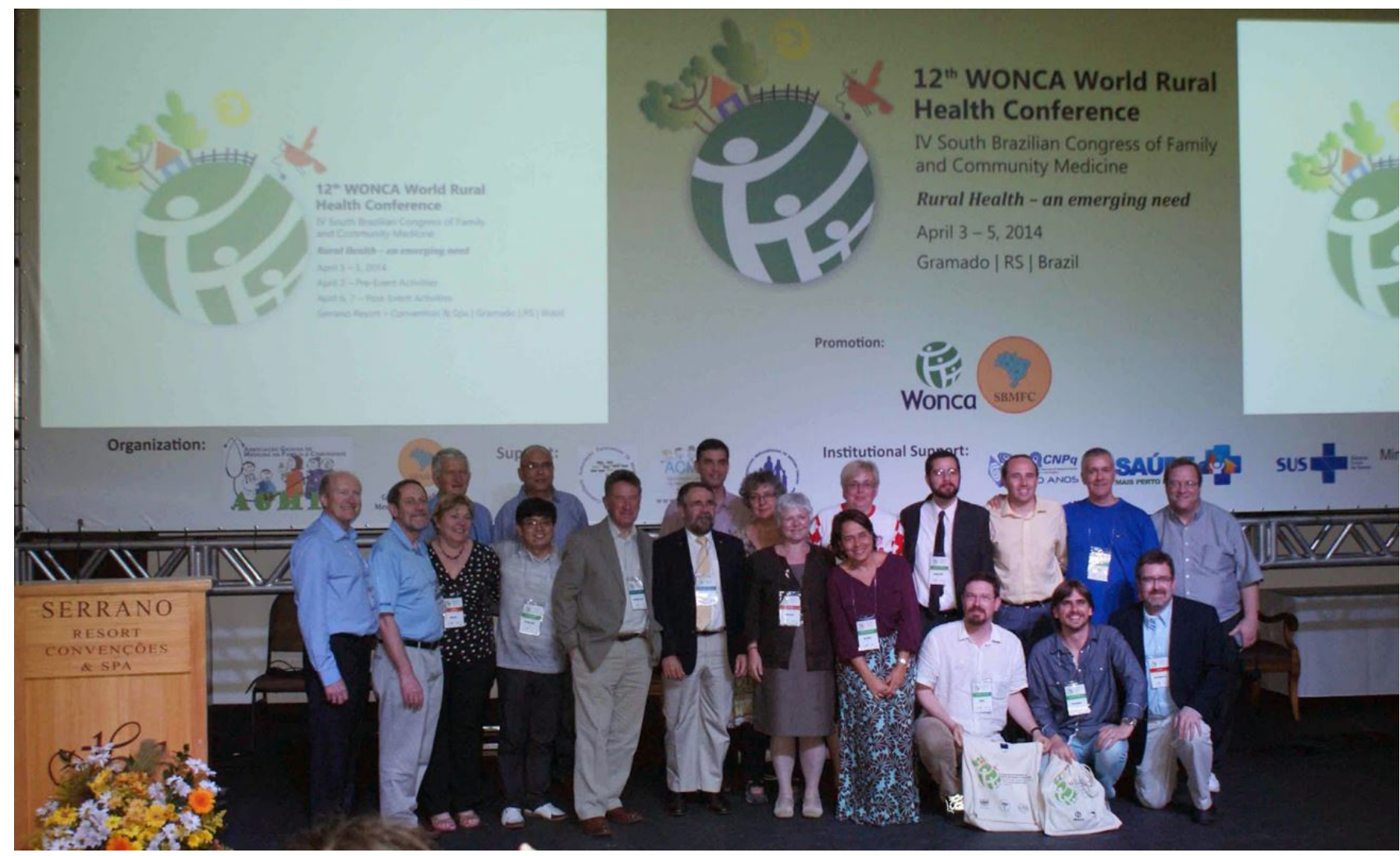

Foto 4. Congresso Mundial de Saúde Rural da WONCA, em Gramado, 2014. Membros do WONCA Working Party on Rural Practice, diretoria da WONCA, diretoria da SBMFC e membros do GT rural participantes do comitê organizador. 
Entre as ações desencadeadas, a partir deste evento, estão o lançamento da seção Latino Americana de um dos principais periódicos científicos internacionais na área, a Rural and Remote Health ${ }^{6}$ e a Declaração de Gramado pela Saúde Rural nos países em desenvolvimento. ${ }^{7}$

Outro aspecto a se destacar como impulsionador dos trabalhos do GT foi o cenário das políticas de saúde no Brasil, em especial a política de recursos humanos. Diante da progressiva organização governamental em torno de uma proposta que posteriormente ficou conhecida como programa "Mais Médicos" e de suas implicações para a saúde das populações rurais e para o espaço de trabalho dos MFCs e das equipes de saúde da família. Foi intenso o trabalho de estudo, reflexão e diálogo em torno do tema, gerando, por fim, um posicionamento oficial da SBMFC, que infelizmente não se concretizou em sua totalidade enquanto política oficial. Entretanto, parece inegável a influência positiva nas propostas governamentais, com intuito de reduzir aspectos negativos do programa, como por exemplo, sua competição com programas de residência e sua inicial falta de intenção fixadora destes profissionais.

A postura do GT rural sempre foi a de tensionar para que o problema da falta de recursos humanos no Brasil fosse alvo de uma política longeva e complexa de formação de profissionais com perfil de MFC, e baseado nas recomendações internacionais e na experiência nacional, ao invés de um programa que inicialmente era pontual e centrado no recrutamento de estrangeiros. A posição oficial do GT, que foi defendida em diversos momentos perante a sociedade e o governo, pode ser conferida na Declaração de Belém, ${ }^{8}$ bem como no Manifesto de Natal, que versou sobre política para fixação da Médica Mulher em área rural, documento que também foi publicado na Revista Brasileira de Medicina de Família e Comunidade. ${ }^{9}$

Outro eixo de trabalho que sempre ocupou muito o GT foi a necessidade de aprimoramento do MFC para trabalhar em áreas rurais, que sabidamente exigem habilidades diferentes do médico e o impõe condições diferenciadas de trabalho. Esta preocupação esteve presente desde o início com a proposta de criação de um R3 (terceiro ano opcional de residência para MFC), que foi apresentada à Comissão Mista de Especialidades da Associação Médica Brasileira já em 2010 e reapresentada posteriormente, visto que não foi obtido o aceite esperado. Posteriormente, estratégias paralelas de inserção de atividades nos programas existentes, criação de convênios para estágio rural opcional e colaboração na elaboração de competências para o médico MFC da SBMFC com enfoque rural foram adotadas. ${ }^{10} \mathrm{~A}$ dificuldade para a implementação de formação específica de R3, com ênfase em áreas rurais, esbarra na ausência de área de concentração específica de medicina rural aprovada pela Comissão Mista de Especialidades, e ao conflito que alguns procedimentos e competências têm gerado com outras áreas e especialidades médicas.

\section{Ativismo de estudantes e jovens médicos brasileiros: a história da Rural Seeds}

Em 2015 foi realizado o primeiro Rural Family Medicine Café, mais conhecido no Brasil como Café Rural. Apesar da ideia ter surgido de uma brasileira, Mayara Floss, a ação não estava inicialmente articulada com o GT Rural, mas sim com integrantes da WWPRP. Porém, isto auxiliou na articulação com o GT nacional e na aproximação de acadêmicos do grupo. A participação inicialmente de outra brasileira foi fundamental para a construção do projeto a Bianca Niemezewski Silveira.

O Café Rural cresceu e em 2016, no WONCA Rio (foto 5 e 6), teve a sua primeira edição com os participantes se encontrando em um palco e não virtualmente, o "café" foi transmitido ao vivo no YouTube 
com interação de pessoas de todo o mundo através do Twitter e Facebook. Atualmente este formato do Café Rural já foi repetido e reproduzido em diversos países, com e sem transmissão ao vivo no YouTube.

Após a criação do Café Rural, em 2016, foi criado o blog "Rural Health Success Stories" (Histórias Rurais de Sucesso) em parceria com a Organização de Médicos de Família Rural do Sul da Ásia (WORSA), coordenado inicialmente por Mayara Floss (Brasil) e Pratyush Kumar (Índia). O blog é um local de compartilhamento de histórias rurais, que tem a potencialidade de reunir os profissionais atuantes em áreas rurais em um espaço para compartilhar suas vivências.

Em 2017, foi criada a "Rural Seeds" (em tradução livre "Sementes Rurais"), uma rede de profissionais em treinamento para trabalhar em áreas rurais. A Rural Seeds foi criada em parceria com a WWPRP que criou também uma cadeira na Executiva Mundial de Saúde Rural com representantes da Rural Seeds. Junto com o lançamento da rede Rural Seeds, na $14^{\text {a }}$ Conferência Mundial de Saúde Rural da WONCA, foi realizada um "Call to Action" (chamada para ação) com a intenção de dar voz à saúde rural a profissionais em treinamento, e para estabelecer metas integradoras e de apoio à esfera da saúde rural, com base nas experiências cumulativas de organizações e indivíduos. Foram levantadas três áreas principais para ação:

- Educação e treinamento;

- Co-empoderamento;

- Comunicação.

Desde então a Rural Seeds tem se concentrado em funcionar com projetos que discutem a saúde rural, com o foco em aumentar o número de profissionais interessados. A saúde rural não pode ser descoberta por acaso e é necessário reduzir o isolamento social e ocupacional dos profissionais de saúde em áreas rurais e remotas.

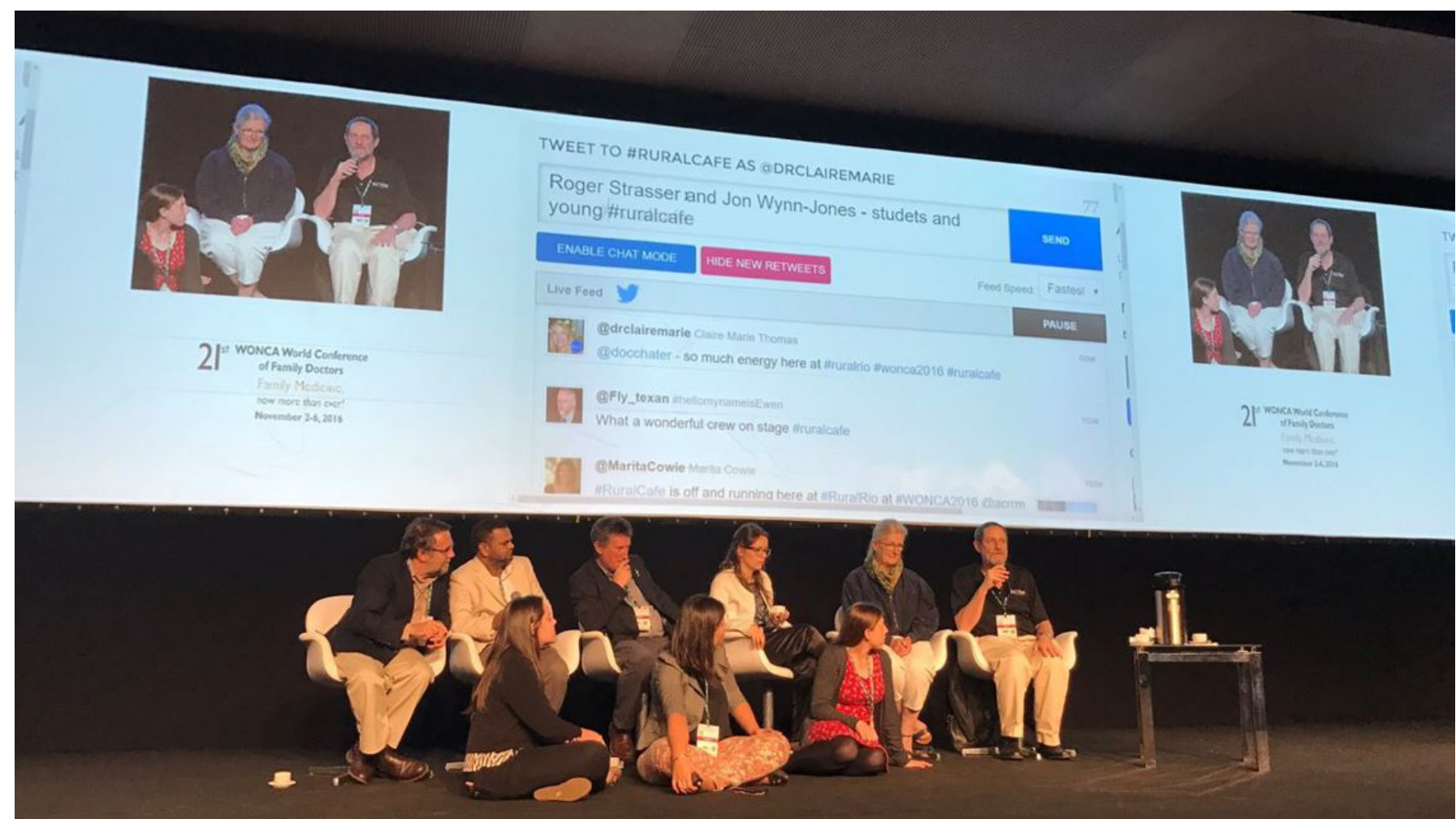

Foto 5.Café Rural com transmissão ao vivo, no WONCA Rio, 2016. Membros do WONCA Working Party on Rural Practice e da Rural Seeds. 


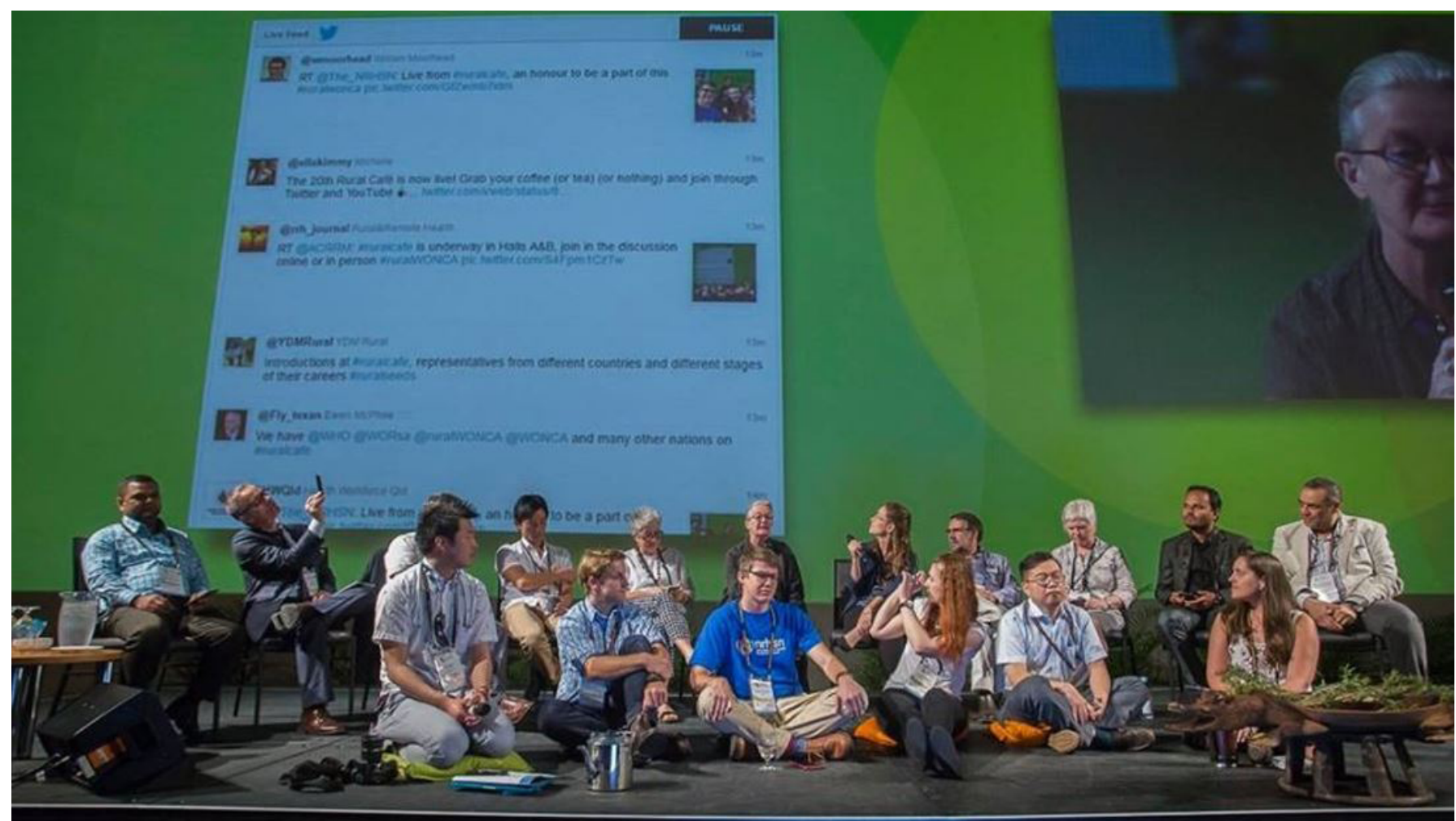

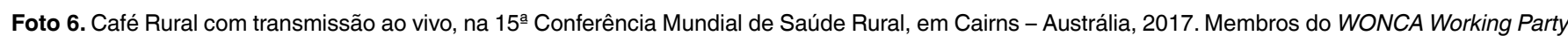
on Rural Practice, Rural Seeds e Organização Mundial de Saúde.

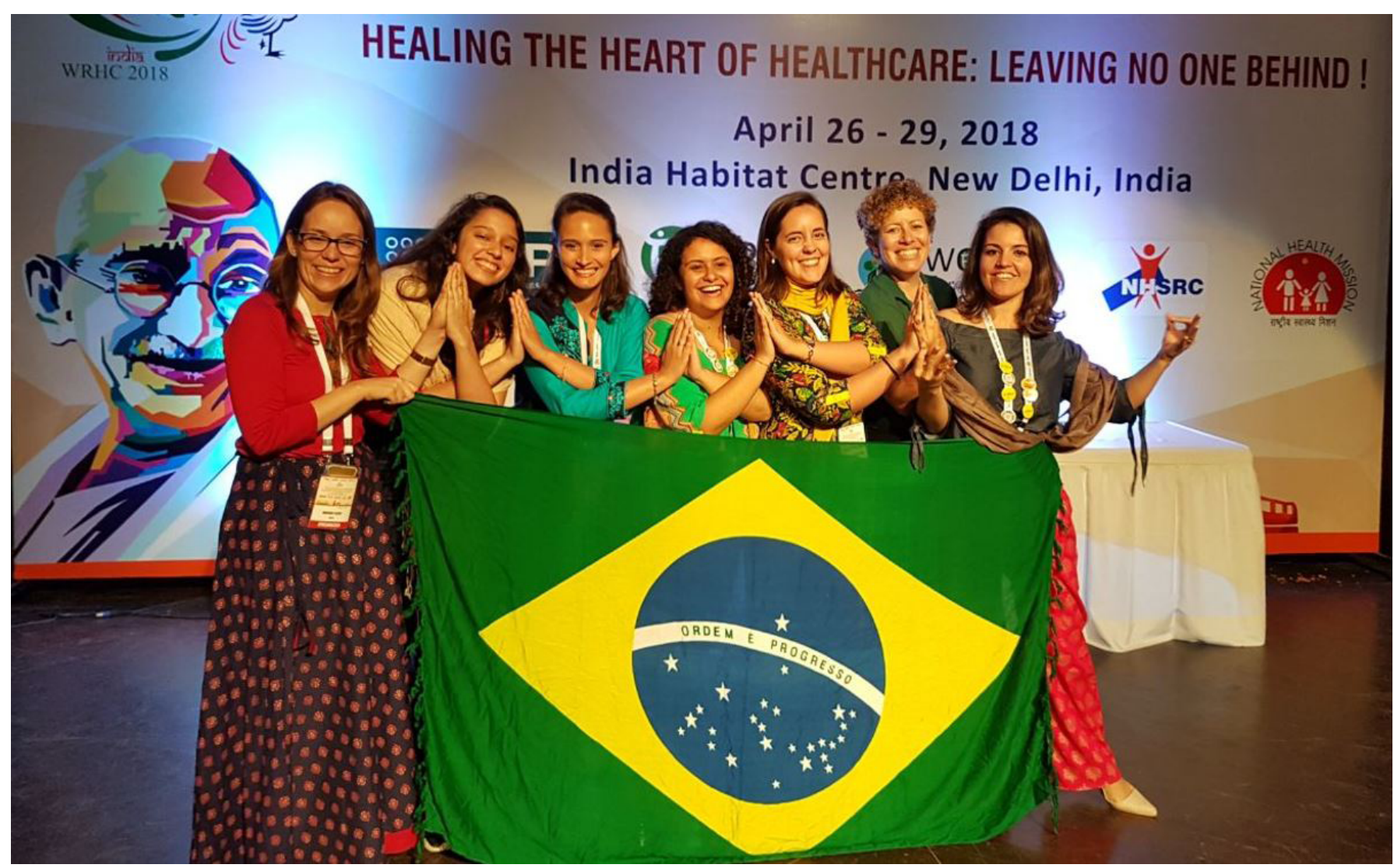

Foto 7. Participação Brasileira na 15ª Conferência Mundial de Saúde Rural, em Nova Déli - Índia. Da esquerda para direita: Mayara Floss, Andressa Paz, Thais Bandeira, Ana Júlia de Araújo, Karine Puls, Magda Almeida e Paola Veiga. 
Em 2017, a Rural Seeds realizou o primeiro projeto de Mentoria Mundial, o "Mentor Mentee", após discussões na Conferência Mundial de Saúde Rural, sobre métodos para reduzir o isolamento dos profissionais de saúde rural. O objetivo do projeto é conectar profissionais médicos rurais em treinamento com colegas mais experientes, para ajudá-los a alcançar seu potencial na área de saúde rural. Desta forma, deve-se reduzir o isolamento e criar acesso mais equitativo às oportunidades de treinamento em todo o mundo. O piloto foi coordenado pela brasileira Mayara Floss teve resultados muito positivos, porém com dificuldades de manter a sua sustentabilidade, o projeto está em análise pelo núcleo da Rural Seeds e, atualmente, é coordenado por Karine Kersting Puls, também médica de família e comunidade rural.

Outro projeto é o "Rural Videos" com o objetivo de explorar alguns tópicos sobre saúde rural, especificamente para profissionais em treinamento. Com o apoio financeiro da Sociedade Brasileira de Medicina de Família e Comunidade (SBMFC), um grupo brasileiro da Rural Seeds conseguiu obter o software e as ferramentas necessárias para produzir vídeos curtos sobre saúde rural, em conjunto com o Grupo de Trabalho Brasileiro para Saúde Rural. Estes também foram utilizados para educar a população em geral sobre tópicos em saúde rural. Diversos vídeos já foram feitos e atualmente o projeto é coordenado pela acadêmica brasileira Andressa Paz.

A participação dos brasileiros na organização da Rural Seeds tem tido um crescimento constante, sendo o grupo brasileiro o mais ativo entre todos os países. Isto tem refletido em um aumento da discussão sobre saúde rural. Também com iniciativas de ligas acadêmicas, que começaram a pautar o tema em suas universidades como o caso da Liga da Saúde - Univates (Lajeado/RS).

Além da discussão em alguns núcleos da Federação Internacional de Associações de Estudantes de Medicina (IFMSA), da Universidade Federal de Uberlândia e da Universidade Federal do Ceará. Também projetos criados e articulados por acadêmicos, como no caso: "Projeto de extensão e projeto de pesquisa: Internato em Saúde Rural: aprendizados e desafios", na Universidade Federal de Uberlândia, coordenado pelas acadêmicas Ana Julia Araújo e Marcela Fontana, sob orientação de Erica Maria Ferreira de Oliveira.

A participação também em eventos regionais, nacionais e internacionais (foto 7) com apresentação de projetos e trabalhos da Rural Seeds tem fomentado a integração dos profissionais em formação. Um exemplo foi uma ação interligas entre a liga da Saúde da Univates (Lajeado/RS) e as ligas da Universidade de Passo Fundo (UPF), e Universidade Federal da Fronteira Sul (UFFS) em Passo Fundo e IMED com as Liga Acadêmica de Iniquidade em Saúde (IMED), Liga Acadêmica de Medicina de Família e Comunidade (UPF) e a Liga Acadêmica de Saúde da Família (UFFS). Esta atividade foi coordenada por duas acadêmicas integrantes da Rural Seeds: Paola Veiga e Andressa Paz, em 2017.

Um grande espaço tem sido também os congressos de educação médica, que com a participação dos integrantes da Rural Seeds, tem trazido a temática de saúde rural para discussão.

Mais informações e links para conhecer a Rural Seeds, além de se inscrever para participar do grupo, podem ser obtidos no site: https://www.ruralseeds.com/. ${ }^{11}$ 
Tabela 1. Linha do tempo da História do GT rural da SBMFC.

2009 - Participação no 17th International Congress of Agricultural Medicine \& Rural Health, Colômbia. Primeiros contatos com WWPRP e articulação para uma representação da América do Sul/Brasil no grupo.

2009 - Participação no 9th WONCA World Rural Health Conference, Grécia. Primeiros contatos com WWPRP.

2009 - Congresso Brasileiro de MFC, Florianópolis. Primeira atividade rural com participação internacional no Brasil e vários temas relacionados à medicina rural na programação oficial.

2010 - Congresso Sul-Brasileiro de MFC, em Curitiba.

2010 - WONCA World Conference, México. Início da representação brasileira no WWPRP e início do alinhamento para a World Rural Health Conference, em Gramado, 2014.

2010 - Primeira proposta de R3 rural apresentada pela SBMFC.

2011 - Início oficial do GT rural da SBMFC, publicação da declaração de Brasília.

2011 - Primeiros capítulos rurais publicados no PROMEF.

2011 - Representação brasileira no Congresso da EURIPA, Romênia.

2011 - Representação no Foro de Expertos en Salud Rural, Argentina. Início de articulações regionais para a conferência mundial, em Gramado, 2014.

2011 - Simpósio Gaúcho de Medicina Rural, Nova Petrópolis.

2011 - Apresentação no congresso ABRASCO e divulgação da conferência de Gramado.

2012 - Representação no Rendez-Vous (Rural Conference WWPRP + The Net), Canadá.

2012 - Congresso Brasileiro de MFC, Rio de Janeiro.

2012 - Congresso Sul-Brasileiro de MFC, Florianópolis.

2012 - IV Congresso Paulista de MFC, Águas de Lindóia.

2012 - Primeiros capítulos rurais no Tratado de MFC (Ceratti e Gusso).

2013 - Capítulos rurais no Medicina Ambulatorial (Bruce Duncan et al.).

2013 - CBMFC, Belém e Declaração de Belém sobre recrutamento e retenção de recursos humanos para áreas rurais.

2013 - Tradução para português dos principais documentos rurais internacionais em número exclusivo rural da RBMFC.

2013 - Apresentação em Seminário da Associação Médica de MG com participação do Ministério da Saúde, Belo Horizonte.

2013 - Apresentação no Seminário Internacional de APS, Porto Alegre.

2013 - Representação e apresentação na WONCA World Conference, Praga.

2014 - Congresso Mundial de Saúde Rural em Gramado e Declaração de Gramado.

2014 - Capítulo no Rural Medical Education Guidebook do WWPRP.

2014 - Abertura da seção latino-americana da Rural and Remote Health.

2014 - V Congresso Paulista de MFC, Ribeirão Preto. I: Mostra de Fotografias de experiências Exitosas

e Fortalecedoras em Medicina Rural. 
2015 - CBMFC, Natal e elaboração do Manifesto de Natal: por uma política afirmativa no recrutamento, formação, provimento, retenção e suporte para as médicas de família em contexto rural e remoto. Publicado posteriormente na RBMFC. Apresentação de Fotos e Vídeos dos Cenários Rurais do Brasil. 2015 - Apresentação e representação no Rural Summit da Sociedade Canadense de Medicina Rural, Montreal.

2015 - Apresentação e representação na WONCA Rural Health Conference, Croácia.

2015 - Participação na elaboração colaborativa de lista de competências do MFC rural pela SBMFC 2015 - Criação do Rural Family Medicine Café.

2016 - Criação do Rural Health Success Stories.

2016 - Congresso Mundial da WONCA, Rio de Janeiro.

2016 - $1^{\circ}$ Café Rural com transmissão ao vivo dos participantes.

2016 - Livro: "Saúde a Caminho da Roça" ganha edital da Coleção Fazer Saúde da FIOCRUZ.

2016 - Capítulo rural no livro de APS da Universidade de Caxias do Sul.

2017 - 14 ${ }^{\circ}$ Congresso Brasileiro de Medicina de Família e Comunidade, em Curitiba. Com exposição de Fotos "As Faces do Rural".

2017 - Lançamento da Rede mundial Rural Seeds, em Cairns - Austrália.

2017 - "Call to action" da Rural Seeds, em Cairns - Australia.

2017 - Lançamento do projeto piloto de Mentoria Mundial -“Mentor Mentee”.

2017 - Lançamento do projeto “Vídeos Rurais” pela Rural Seeds.

2017 - Seminário Internacional de Saúde Planetária, Porto Alegre, com inserção de apresentação relacionando impacto rural.

2017 - Capítulos rurais na $2^{a}$ edição do Tratado MFC.

2017- Por que saúde rural? TEDxUnisinos (https://www.youtube.com/watch?v=H7VewJXVLE0).

2018 - It is time for rural training in family medicine in Brazil!: editorial na RBMFC.

2018 - Congresso Mundial de Saúde Rural na Índia - Mayara recebe o Health Leadership Award.

2018 - Premiação de melhor animação do FISFA - International Short Film and Arts Festival, do projeto dos vídeos rurais com o vídeo "Porque a Saúde Rural".

2018 - Publicação do Livro Saúde no Caminho da Roça - livro FIOCRUZ.

2019 - Participação no Congresso Gaúcho de Educação Médica, em Passo Fundo.

2019 - Congresso Brasileiro de Medicina de Família e Comunidade, em Cuiabá.

2019 - Premiação de melhor vídeo em primeiro lugar com o projeto Vídeos Rurais o vídeo "Francesa Alta" e em segundo lugar "Quem é e o que faz o Médico de Família e Comunidade Rural?".

2019 - WONCA Rural Health Conference, EUA.

2019 - AAFP Global Health Summit, Albuquerque - New Mexico.

2019 - Participação no Ourense Rural Health Summit, Espanha. 
Outros documentos foram produzidos pelo GT nos anos seguintes, acompanhando os Congresso Brasileiros de MFC. A inserção de aulas e estágios em saúde rural em cursos de graduação e residência de medicina de família foi outro passo estratégico, bem como a inclusão de capítulos rurais em livros de referência para a área. $\mathrm{O}$ envolvimento de jovens médicos e residentes nos últimos anos tem sido um dos grandes motores do GT.

Conclui-se, ao revisar a história do GT rural da SBMFC, que muitos colegas se envolveram com sucesso no trabalho de criar uma identidade, a do "MFC rural", e de trazer a discussão da saúde das populações rurais para a pauta dos meios da saúde, da atenção primária e da medicina de família. Para tanto foram vitais a articulação com o movimento mundial de médicos rurais, o apoio da SBMFC e de várias de suas regionais, o envolvimento de jovens médicos e estudantes e o cenário político em que as questões de recursos humanos para a saúde tem estado em pauta. Evidentemente, o sucesso de toda esta história sempre esteve baseado em pessoas apaixonadas e dispostas a trabalhar muito pessoal e coletivamente pela saúde das populações rurais e pela medicina de família.

\section{REFERÊNCIAS}

1. Sociedade Brasileira de Medicina de Família e Comunidade (SBMFC). Regulamentação dos Grupos de Trabalho da SBMFC [Internet]. Rio de Janeiro (RJ): SBMFC; 2017; [citado 2019 ago 4]. Disponível em: http://www.sbmfc.org.br/wp-content/uploads/media/file/ REGIMENTO\%20GTS_2018.pdf

2. Targa LV. A avaliação da pressão arterial por agentes comunitários pode ser uma estratégia útil para o cuidado da saúde?.Rev Bras Med Fam Comunidade [Internet]. 2006Nov; [citado 2020 janeiro 18];1(4):141-51. Disponível em: https://www.rbmfc.org.br/rbmfc/article/view/46 DOI: https://doi.org/10.5712/rbmfc1(4)46

3. Ando NM, Targa LV, Almeida A, Silva DHS, Barros EF, Schwalm FD, et al.DECLARAÇÃO DE BRASíLIA"O Conceito de rural e o cuidado à saúde". RevBrasMedFamComunidade. 2011;6(19):142-4. DOI: https://doi.org/10.5712/rbmfc6(19)390 DOI: https://doi.org/10.5712/ rbmfc6(19)390

4. Targa LV, Ando NM, Silva AL, Silveira RP, Porto MMA, Amaral Filho RCG, et al. As recomendações da Wonca para a Saúde das Populações Rurais. Rev Bras Med Fam Comunidade [Internet]. 2013; [citado 2020 jan 18];8(1):4-6. Disponível em: https://www.rbmfc.org.br/rbmfc/ article/view/724

5. Duncan MS, Targa LV. Médicos para atenção primária em regiões rurais e remotas no Brasil: situação atual e perspectivas. Rev Bras Med Fam Comunidade [Internet]. 2014 Set; [citado 2020 jan 18]; 9(32):233-4. Disponível em: https://www.rbmfc.org.br/rbmfc/article/view/1004

6. Targa LV, Couper I, Worley P. A new ally for the promotion of better rural health in Latin America. Rural Remote Health. 2014;14(4):3376.

7. Targa LV, Wynn-Jones J, Howe A, Anderson MIP, Lopes JMC, Lermen Junior N, et al. Declaração de Gramado pela Saúde Rural nos países em desenvolvimento. Rev Bras Med Fam Comunidade [Internet]. 2014Jul; [citado 2020 jan 18];9(32):292-4. Disponível em: https:// www.rbmfc.org.br/rbmfc/article/view/982

8. Targa LV, Silva AL, Silva DHS, Barros EF, Schwalm FD, Savassi LCM, et al. Declaração de Belém: o recrutamento e a retenção de profissionais de saúde em áreas rurais e remotas. Rev Bras Med Fam Comunidade [Internet].2014Jan/Mar; [citado 2020 jan 18]; 9(30):64-6. Disponível em: https://www.rbmfc.org.br/rbmfc/article/view/827 DOI: https://doi.org/10.5712/rbmfc9(32)982

9. Lima MC, Cuadrado M, Horta TCG, Almeida M, Pinheiro A, Maia PCGGS, et al. Manifesto de Natal - Por uma política afirmativa no recrutamento, formação, provimento, retenção e suporte para as médicas de família em contexto rural e remoto. Rev Bras Med Fam Comunidade [Internet]. 2016 Set; [citado 2020 jan 18];11(38):1-5. Disponível em: https://www.rbmfc.org.br/rbmfc/article/view/1247

10. Sociedade Brasileira de Medicina de Família e Comunidade (SBMFC). Matriz de Competências de MFC é publicada pelo MEC [Internet]. Rio de Janeiro (RJ): SBMFC; 2019; [citado 2019 ago 4]. Disponível em:https://www.sbmfc.org.br/noticias/matriz-de-competencias-de-mfc/

11. Rural Seeds [homepage]. WONCA Rural Health; 2017; [citado 2019 ago 4]. Disponível em: https://www.ruralseeds.com/ 